\title{
QUALITY OF SINGLE CONE AND GUTTA CORE OBTURATION IN CURVED ROOT CANALS AFTER NEEDLE OR DIODE LASER IRRIGANT ACTIVATION USING CONE BEAM COMPUTED TOMOGRAPHY AND STEREOMICROSCOPE
}

\author{
Latifa Mohamed Abdelgawad ${ }^{*}$
}

\begin{abstract}
Objective: The purpose of this study was to assess the quality of single cone and gutta core obturation in curved root canals of the mandibular molars after needle and diode laser activation of both ethylenediaminetetraacidic acid (EDTA) and sodium hypochlorite $(\mathrm{NaOCl})$ irrigation using cone beam computed tomography (CBCT) and stereomicroscope.

Methods: A total of twenty -four extracted human mandibular molars with root canal curvature more than 20 degrees and type IV anatomic configuration in the mesial roots were selected for this study. Canals in mesial roots were prepared with ProTaper next instruments up to x 3 and equally divided into two experimental groups $(\mathrm{n}=12)$ according to final one minute activation for each of $5 \mathrm{ml} \mathrm{2.5 \%} \mathrm{NaOCl}$ and $5 \mathrm{ml} \mathrm{15 \%}$ EDTA using; side-vented needle (group1) or $980 \mathrm{~nm}$ diode laser (group2). After preparation, the mesial canals were randomly obturated with either single-cone gutta-percha (sub group A) or gutta core Obturator (subgroup B). Obturated canals were scanned with cone beam computed tomography; images were used to assess the quality of obturation at different canals levels. The roots were subsequently sectioned at 2, 4, 6, 8 and $12 \mathrm{~mm}$ levels from the apex for analyses of the percentage of gutta percha filled area (PGFA) and percentage of sealer filled area (PSFA) using stereomicroscope to examine the root sections. The areas of gutta percha and sealer for each segment of the root were measured using Image J software. Kruskal-Wallis test was used to analyze data.
\end{abstract}

Results: Both cone beam and digital stereomicroscopic data indicated that canals obturated with gutta core after irrigant activation had the highest PGFA. The Gutta core and diode laser activation group produced significantly higher PGFA and lower PSFA at different root canal levels 4,6 and $8 \mathrm{~mm}$ levels of the root canal $(\mathrm{P}<.05)$ than all other groups at the same levels $(\mathrm{P}<.05)$. Single cone and EDTA has the lowest PGFA and highest PSFA $(\mathrm{P}<.05)$.

Conclusions: The results reinforced that $980-\mathrm{nm}$ Diode activated irrigation improved the quality of obturation of the mesial root canals in mandibular molars by using gutta core or single cone. Variation in root canal obturation technique and irrigation activation influenced the percentage of gutta percha filled area and percentage of sealer filled area.

KEYWORDS: Diode laser, side-vented needle, gutta-percha filled area, sealer filled area, curved root canals mandibular Molars, CBCT and stereomicroscope.

* Associate Professor in Endodontics, Medical Applications Department, National Institute of Laser Enhanced Sciences NILES, Cairo University, Egypt. 


\section{INTRODUCTION}

Three dimensional obturation of the root canal space is essential step in root canal treatment with the goal to maximize the amount of solid core material and minimize the amount of sealer ${ }^{(1)}$.

A proper chemomechanical preparation of the root canal system and removal of the smear layer is recommended prior to obturation ${ }^{(2,3)}$ However, irrigating solutions can be inefficient in removing the smear layer from the apical thirds of root canals ${ }^{(3,4)}$, while improved irrigant activation methods can overcome this problem ${ }^{(5-10)}$.

Recently, agitation of irrigating solutions with laser devices has become popular ${ }^{(11-13)}$.Some studies evaluated the role of using Diode laser activated irrigation in smear layer and debris removal ${ }^{(14-16)}$.

After chemomechanical preparation different methods used for root canal obturation include cold lateral compaction, single-cone gutta-percha, and Warm vertical compaction and core-carrier systems ${ }^{(17,18)}$.

Filling root canals by lateral condensation of gutta percha traditionally uses a master .02 taper standard gutta-percha cone followed by the addition of further accessory standard gutta-percha cones after lateral condensation of the gutta-percha with spreaders. This process is time consuming and has the potential to place undue force on the root leading to root fracture ${ }^{(17) .}$

The use of single-cone gutta-percha matching the taper and diameter of the canals prepared with engine-driven nickel-titanium (NiTi) instruments, these cones may provide three-dimensional obturation of the root canal over its whole length without the requirement for accessory cones or time spent on lateral condensation ${ }^{(1)}$.

Core-carrier systems consisted of metal and later plastic carriers that are coated with alpha-phase gutta-percha. Obturation with these systems provides a $3 \mathrm{D}$ obturation and ensures adequate filling of canal irregularities and flow of the gutta-percha into lat- eral canals ${ }^{(19)}$, and thus a maximum amount of gutta percha packed into the canal with a minimal amount of sealer. This aspect is of clinical relevance because gutta percha is dimensionally stable ${ }^{(20)}$.

Recently, 2 new core-carrier systems were introduced: GuttaCore (GC) (Dentsply Maillefer, Ballaigues, Switzerland) and GuttaFusion (GF) (VDW, Munich, Germany). The core of these systems consists of cross-linked thermoset guttapercha that does not melt at temperatures generated by the special oven ${ }^{(21)}$

Some studies have assessed the quality of these single-cone fillings regarding sealing ability ${ }^{(9,13-20)}$, and percentage of gutta-percha and sealer-filled canal area ${ }^{(6-8,10,22,23)}$. In general, most studies reported similar results obtained with single-cone obturation compared with the lateral compaction technique or methods that used themoplasticized gutta-percha (7-9,14-19, 21, 22, 24), whereas in other reports, single-cone obturation was found to result in

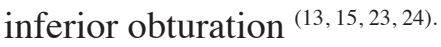

However No attempts have been made to assess the quality of single cone and gutta core obturation after diode laser activation using cone beam. Therefore, the purpose of this study was to assess the quality of single cone and gutta core after needle and diode laser activation techniques using cone beam and stereomicroscope.

\section{MATERIALS AND METHODS}

\section{Selection of samples}

A total of twenty -four extracted human mandibular molars with root canalcurvature more than 20 degrees as determined by the method of Schnneider ${ }^{25)}$ and type IV anatomic configuration (two separate root canals from orifice to apical foramen, according to Vertucci's classification ${ }^{(26)}$ and similar length $19 \pm 1 \mathrm{~mm}$ were selected for this study . The teeth were extracted for periodontal reasons and carefully examined for the absence of cracks, resorption, root caries or previous root canal treat- 
ment.The teeth were disinfected then kept in distilled water until used.

\section{Preparation of samples}

After access cavity preparation,the distal roots of the teeth were removed completely.A \# $10 \mathrm{~K}$-file was inserted into the root canal until its tip was just visible at the apical foramen and the working length (WL) was established one mm short of measured length. One operator instrumented the root canals with Path files and ProTaper Next system until X3 (Dentsply,Maillefer, Ballaigues, Switzerland), using X-Smart motor at $300 \mathrm{rpm}, 2 \mathrm{Ncm}$ (Dentsply, Switzerland). At every instrument change, the canals were irrigated with $5 \mathrm{ml} 2.5 \% \mathrm{NaOCl}$ solution (Clorox, Nobelwax Factories for chemicals, Egypt). A 5ml- syringe with $30-\mathrm{G}$ side-vented needle (Canal Clean, Biodent, Co, Ltd, Pagu City, Korea) was inserted $2 \mathrm{~mm}$ short of the WL.

\section{Final irrigation protocols}

After the chemomechanical preparation, the mesial roots were randomly divided into two equal groups according to final activated irrigation protocols; either by side-vented needle (group1) or diode Laser (group 2). Final irrigant activation was performed for $5 \mathrm{ml} 2.5 \% \mathrm{NaOCl}$ and $5 \mathrm{ml} 15 \%$ EDTA (Endo-Solution, Cerkamed, StalowaWola, Polska). Activation was only done for EDTAand $\mathrm{NaOCl}$ according to the following sequence: activation of $1 \mathrm{ml}$ EDTA $15 \%$ (12 sec), $1 \mathrm{ml}$ distilled water, activation of $1 \mathrm{ml} \mathrm{NaOCl} 2.5 \%$ (12 sec), finally 1 $\mathrm{ml}$ distilled water. The sequence was repeated five times, giving $5 \mathrm{ml}$ total volume and $60 \mathrm{sec}$ total.

\section{The activation mechanisms in the two groups were as follows;}

Group 1(N group): side-vented needle delivered the solutions while moving slowly up and down along canal length. All activations were performed such that the maximum depth was $2 \mathrm{~mm}$ away from WL.

Group 2 (L group): A continuous wave diode Laser with 980-nm wavelength and average power
2 W (Quanta Laser systems, Italy) was used for irradiation. The laser was delivered into $320 \mu \mathrm{m}$ flexible plain endodontic fiber. The fiber was used with constant motion in apical-coronal direction and kept $3 \mathrm{~mm}$ away from the root apex.

\section{Obturation of the root canals}

Group 1 and group 2 will be subdivided into two equal subgroups according to obturation; subgroup A; obturated by single cone (S C) ProTaper next gutta percha X3 (Dentsply Maillefer, Ballaigues, Switzerland); sub group B, obturated by gutta core (G C) obturator X3 (Dentsply Maillefer, Ballaigues, Switzerland).

In all groups, the root canals were dried with absorbent paper points (Dentsply Maillefer, Ballaigues, Switzerland) and Topseal sealer (Dentsply Tulsa Dental Specialties, Switzerland), was applied before obturation by using a finger spreader size 30 in a counter clockkwise rotation.

\section{Single cone obturation}

A single cone ProTaper gutta percha next size X3 was use to obturate the root canals in this group. The cone was then lightly coated in sealer and placed slowly into the full working length. The excess coronal gutta-percha was removed by a heated instrument (12).

\section{Gutta core obturation.}

Root canals were obturated by using the size X3 Gutta Core Obturator according to the directions specified by the manufacturer. The fitting of the obturator was checked with corresponding verifier. Core obturators were heated in Thermaprep plus oven ((Dentsply Maillefer, Holland). While the canal was coated with the root canal sealer. The heated guttaCore Obturator was slowly inserted to working length without twisting or forcing. The obturator was severed at canal orifice after cooling the gutta percha [1].

After obturation, the teeth were digitally radiographed (Trophy Radiology, 94300 Vin Cennes, 
Type 6510 , France) to confirm the adequacy of root fillings. All roots were coded and stored for 14 days at $37^{\circ} \mathrm{C}$ and $100 \%$ humidity to allow the sealer to set completely.

\section{Evaluation of obturation by cone beam comput- ed tomography (CBCT)}

All samples submitted for CBCT for evaluation of root canal obturation. CBCT images were acquired using a Next Generation i-CAT scanner (Imaging Sciences International, Inc., Hatfield, USA). A scout view was obtained and adjustments were made to ensure that all samples were correctly aligned in the scanner according to adjustment light beam before acquisition. The machine is supplied with Amorphous Silicon Flat Panel Sensor with Cesium Iodide (CsI) scintillator, $0.5 \mathrm{~mm}$ focal spot size, 14 Bit gray scale resolution, and operating at the following protocol for all the scans of the study: tube voltage, $120 \mathrm{kVp}$; tube current, $37.07 \mathrm{~mA}$; scanning time 26.9 seconds; voxel size, $0.125 \mathrm{~mm}$; and field of view $4 \mathrm{~cm}$ height and $16 \mathrm{~cm}$ diameter. After acquisition, data were exported and transferred in DICOM format and downloaded via a Compact Disk (CD) to a personal computer for analysis, where, In vivo Dental software (version 5.1; Anatomage, San Jose, CA, USA) was utilized for angular and linear measurement, while mimics software (materialize, Belgium) was used for volume measurement.

\section{Evaluation of obturation by Stereomicroscope}

Sections were made using a diamond disc (KG Sorensen) with copious cold running water to minimize distortion of the root canal filling. Sections were made at 2, 4, 6, 8 and 12 from the apex of the roots. The sections were viewed under a stereomicroscope camera at $130 \mathrm{X}$ magnification (Microscope camera; Sharper image, china). Digitized images were analyzed using Image $\mathbf{J}$ software (National Institutes of health, public domain) to calculate the percentage ratios of gutta-percha, sealer to the total root canal area (Schäfer E et al., 2016).

\section{Statistical analysis}

Overall influence of each activation protocol of irrigation and root canal filling techniques on both percentages of gutta-percha filled area (PGFA) and sealer filled area (PSFA) were analyzed using Kruskal-Wallis test. The level of significance was set at $\mathrm{P}<.05$.

\section{RESULTS}

\section{A) Cone beam results}

The CBCT images of the obturated tooth are shown in coronal, sagittal, axial and 3D view [Fig 1, Fig-2]

\section{B) Stereomicroscope}

The results for percentage of gutta percha filled area (PGFA) and percentage of sealer filled area PSFA at 2, 4, 6, 8 and $12 \mathrm{~mm}$ from the apex in each group: table 1, Figure 3.

At the 2-mm level, the Gutta core and diode laser group produced significantly higher PGFA and lower PSFA $(\mathrm{P}<.05)$ than all other groups. The gutta core and needle group produced significantly higher PGFA than Single cone and needle and Single cone and diode laser group $(\mathrm{P}<.05)$.

At the 4-mm level, the Gutta core and diode laser group produced significantly the highest PGFA and lowest PSFA $(\mathrm{P}<.05)$ and Single cone and needle has the lowest PGFA $(\mathrm{P}<.05)$. There was no significant difference between Gutta core and needle group and Single cone and diode laser ( $\mathrm{P}>.05)$.

At the 6-mm level, the Gutta core and diode laser group produced significantly higher PGFA and lower PSFA $(\mathrm{P}<.05)$ than all other groups. The Single cone and diode laser produced significantly higher PGFA than Gutta core and needle group and Single cone and needle. Single cone and needle has the lowest PGFA $(\mathrm{P}<.05)$.

At the 8-mm level, the Gutta core and diode laser group produced significantly the 
highest PGFA $(\mathrm{P}<.05)$. Whereas no significant differences between Gutta core and needle group and Single cone and diode laser $(\mathrm{P}>$.05). Single cone and needle has the lowest PGFA $(\mathrm{P}<.05)$. There was no significant difference between group Gutta core and needle group and Single cone and diode laser. $(\mathrm{P}>.05)$.
At the 12-mm level, as regard PGFA, no significant differences were found between Single cone and needle and Single cone and diode laser $(\mathrm{P}>.05)$ ,also no significant differences were found between Gutta core and needle and Gutta core and diode laser group $(\mathrm{P}>.05)$.

TABLE (1) Mean and standard deviation of the percentage of gutta -percha filled area (PGFA) and percentage of sealer filled area (PSFA) in different groups at different levels

\begin{tabular}{|c|c|c|c|c|c|}
\hline \multirow{2}{*}{ level } & filling & S C and needle & S C and L & G C and needle & G C and L \\
\hline \multirow{3}{*}{$2 \mathrm{~mm}$} & PGFA & $71 \pm 12.7^{\mathrm{a}}{ }_{\mathrm{A}}$ & $84.6 \pm 3.13^{\mathrm{b}}$ & $94.34 \pm 3.6^{\mathrm{c}}$ & $90.6^{\mathrm{b}} 3.6^{\mathrm{c}}{ }_{\mathrm{AB}}$ \\
\cline { 2 - 6 } & PSFA & $29 \pm 12.6^{\mathrm{a}}$ & $15.4 \pm 3.13^{\mathrm{b}}$ & $5.742 \pm 3.5^{\mathrm{c}}$ & $9.57+3.3^{\mathrm{c}}$ \\
\hline \multirow{3}{*}{$4 \mathrm{~mm}$} & PGFA & $79 \pm 5^{\mathrm{a}}{ }_{\mathrm{A}}$ & $89.8 \pm 2.9^{\mathrm{b}}$ & $89.8 \pm 6.3^{\mathrm{C}}$ & $92.5 \pm 4.2^{\mathrm{C}}$ \\
\cline { 2 - 6 } & PSFA & $21 \pm 5^{\mathrm{a}}$ & $10.2 \pm 2.9^{\mathrm{b}}$ & $10.2 . \pm 3.4^{\mathrm{b}}$ & $7.5+4.2^{\mathrm{b}}$ \\
\hline \multirow{3}{*}{$6 \mathrm{~mm}$} & PGFA & $83.02 \pm 6.5^{\mathrm{a}}$ & $91.8 \pm 3.4^{\mathrm{b}}$ & $90.8 \pm 7.4^{\mathrm{b}}$ & $93.4 \pm 4.3^{\mathrm{b}}{ }_{A B}$ \\
\cline { 2 - 6 } & PSFA & $16.98 \pm 3.395^{\mathrm{a}}$ & $8.2 \pm 3.397^{\mathrm{b}}$ & $9.2 \pm 1.8^{\mathrm{b}}$ & $6.6+4.2^{\mathrm{c}}$ \\
\hline \multirow{3}{*}{$8 \mathrm{~mm}$} & PGFA & $83.7 \pm 8.4^{\mathrm{a}}{ }_{\mathrm{B}}$ & $94.07 \pm 2.2^{\mathrm{b}}$ & $92.3 \pm 5^{\mathrm{b}}$ & $97.2 \pm 2.04^{\mathrm{c}}{ }_{\mathrm{AB}}$ \\
\cline { 2 - 6 } & PSFA & $12.33 \pm 4.8^{\mathrm{a}}$ & $5.93 \pm 2.2^{\mathrm{b}}$ & $7.7 \pm 5^{\mathrm{b}}$ & $2.8 \pm 2.04$ \\
\hline \multirow{2}{*}{$12 \mathrm{~mm}$} & GP & $85.3 \pm 4.3^{\mathrm{a}}{ }_{\mathrm{B}}$ & $86 \pm 2^{\mathrm{a}}$ & $93.2 \pm 6.1^{\mathrm{b}}$ & $91.14 \pm 3.2^{\mathrm{b}}$ \\
\cline { 2 - 6 } & PSFA & $13.79 \pm 3.8^{\mathrm{a}}$ & $13.6 \pm 1.9^{\mathrm{a}}$ & $6.86 \pm 1.3^{\mathrm{b}}$ & $8.86 \pm 3.2^{\mathrm{b}}$ \\
\hline
\end{tabular}

Horizontally: Different Small letters indicate significant difference $(p<0.05)$ by comparing the same level in different groups. Vertically: Different Capital letters indicate significant difference $(p<0.05)$ by comparing different levels

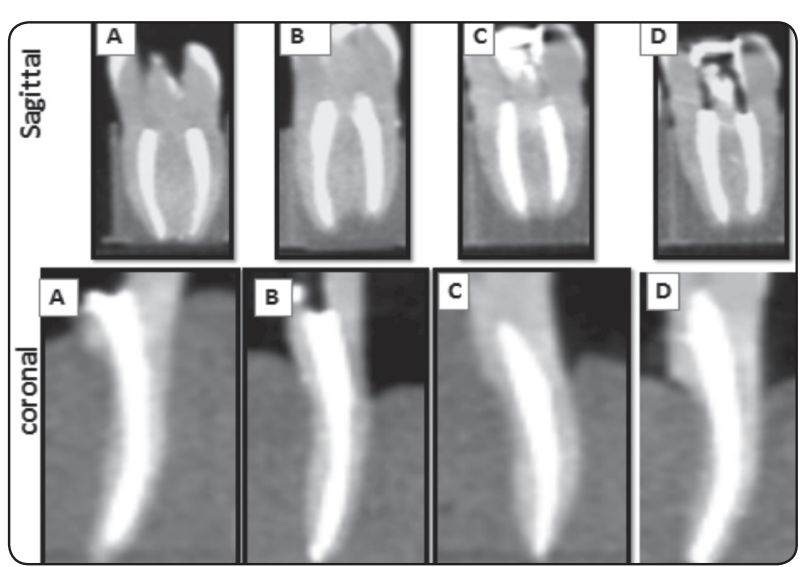

Fig. (1) Cone beam sagittal and coronal images of mesial roots of mandibular molars after needle $(\mathrm{N})$ and diode laser (L) activation and obturation with single cone (S C) or gutta core (G C) ;(A, C) after activation with Side-vented needle, (B, D) after 980-nm Diode laseractivation.

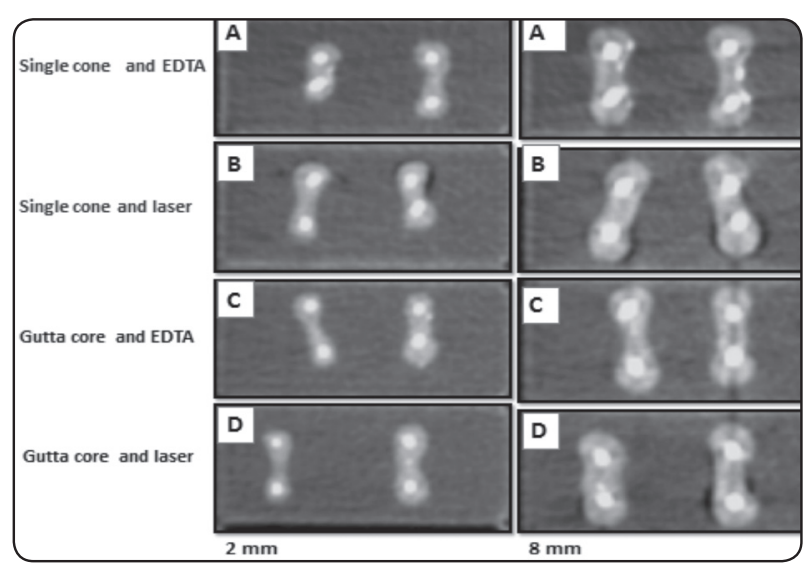

Fig. (2) Cone beam axial images of mesial roots of mandibular molars after needle $(\mathrm{N})$ and diode laser (L) activation and obturation with single cone (S C) or gutta core (G C) ; (A, C) after activation with Side-vented needle, (B, D) after 980-nm Diode laseractivation at 2 and 8 mm levels . 


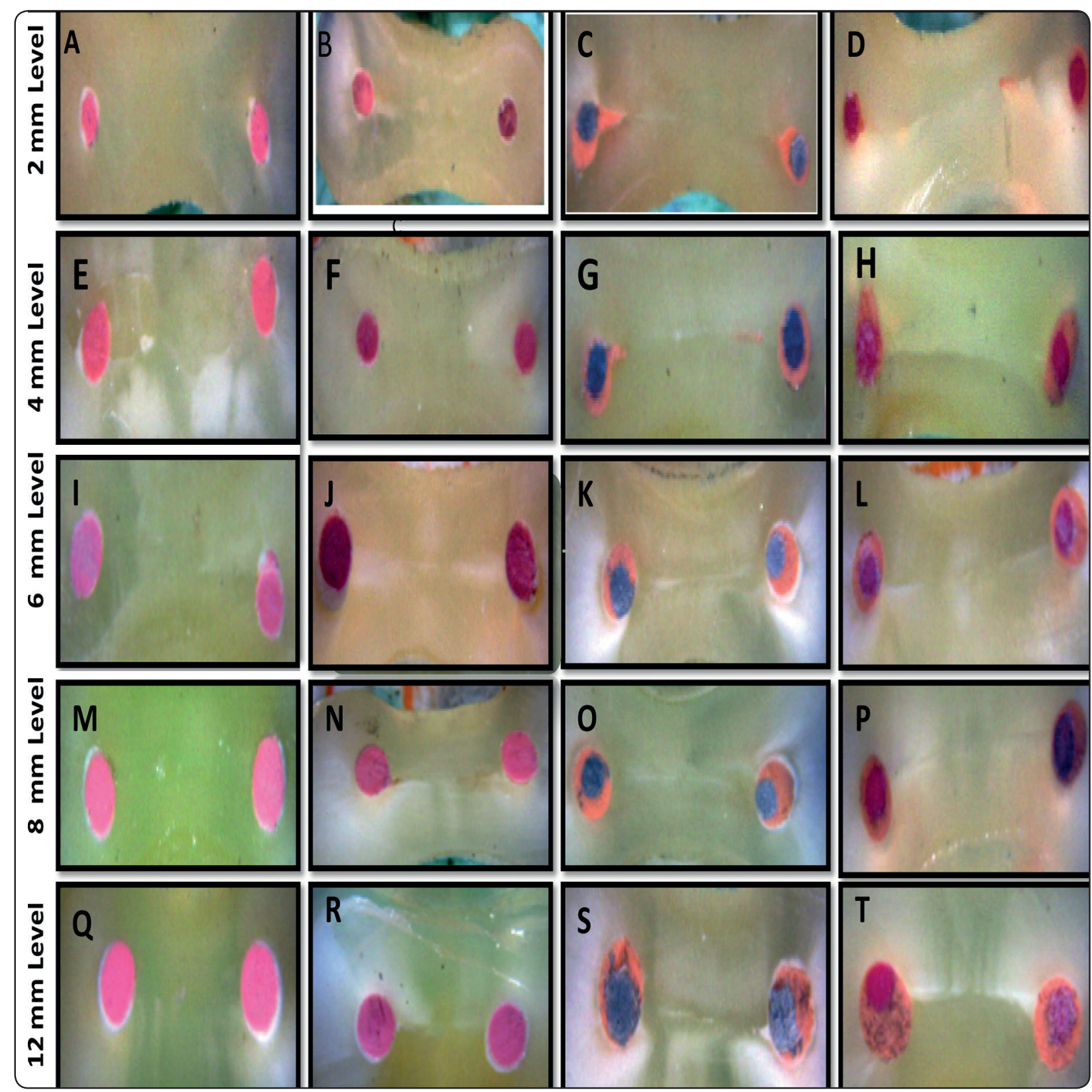

Fig .(3) Panel representative photomicrographs showing different canal levels; $2,4,6$, 8 and 12 mm from the apex (original magnification , $130 \mathrm{X}$ ) after irrigant activation with Side-vented needle $(\mathrm{N})$ or 980-nm Diode laser (L) and root canal obturation with single cone $(\mathrm{S} \mathrm{C})$ or gutta core $(\mathrm{G} \mathrm{C})$. Images on first Colum represent $(\mathrm{SC}+\mathrm{N})$, second Colum represent $(\mathrm{SC}+\mathrm{L})$. , Third Colum represent $(\mathrm{GC}+\mathrm{N})$, fourth Colum represent $(\mathrm{GC}+\mathrm{L}))$. 


\section{DISSCUSION}

To enhance the dispersal of the irrigant and to transport it into the apical parts of the curved root canals, manual and machine -assisted activation techniques have been developed and investigated for smear layer removal (27). Laser activated irrigation recently introduced and developed for this purpose with wavelength from 940-2940 nm 28). Laser activated irrigation is based on cavitation induced by high absorption of water or canal fluid by infrared wavelength of lasers, the cavitation process generates vapor- containing bubbles, which explode and implode in a liquid environment. This initiates pressure /shock waves by inducing the shear force on dentinal wall, which could potentially detach the smear layer ${ }^{(27)}$.

In the present study, laser fiber tip was applied 3 mm away from the apex with the lower power setting $(2 \mathrm{w})$. The risk of extrusion is expected to be lower for A $980 \mathrm{~nm}$ diode laser because it is less absorbed by water compared with higher wavelength erbium lasers .In this respect Matsuoka et al (29) suggested that 200 or 300 um fiber of $2740 \mathrm{~nm} \mathrm{Er,Cr:YSGG}$ laser with 2 watts should be kept 2 and $3 \mathrm{~mm}$ away from the anatomic apex to prevent destruction of the apical constriction .

When the laser is used for intracanal applications, thermal damage to surrounding periodontal tissue is of major concern. To resolve this issue in the present study, laser irradiation is limited to the fiber tip with repeated resting time between the laser irradiation, circular movement of the laser fiber in the root canal and frequent rinsing assisted in cooling, this was supported by other studies ${ }^{(16,30)}$.

This study did not observe directly the effect of needle or diode laser on the removal of smear layer. Rather, the cone beam and stereomicroscopic analysis of the obturation quality for single cone and gutta core in curved root canals, as it can be a good indicator for evaluating the degree of smear layer removal in vitro in tooth.
Proper obturation of the enlarged and cleaned root canal aims at packing a maximum amount of gutta-percha into the canal and keeping the amount of sealer to a minimum because most sealers shrink on setting and dissolve over time ${ }^{(24,31)}$, whereas gutta-percha is dimensionally stable ${ }^{(17)}$. Therefore, the quality of obturation as well as PGFA and PSFA has been used to evaluate the quality of obturation in the present investigation, as was done before in other studies $(18,21,22)$.

In the present study, two obturation techniques were used; The single-cone technique did not use compaction forces and gutta core technique is claimed to enhance adaptation of the gutta percha to the canal wall, and flow of the filling material to fill the canal irregularities and lateral canals ${ }^{(21)}$.

Different methods have been utilized for examination of filling materials inside the root canals, including destructive and non-destructive methods. Destructive methods involve sectioning of the filled root canals and examination by light microscopy, confocal laser scanning electron microscopy or scanning electron microscopy $(\mathrm{SEM})^{(27,31)}$. Non-destructive methods, including cone beam and Micro-computed tomography (micro-CT) $^{(32,33)}$

In the present study, non-destructive and destructive techniques were used to evaluate obturation. Cone beam is non-destructive technique as the specimens remain intact during examination and may be subsequently sectioned and examined by stereomicroscope. Also it provides rapid imaging and 3-D visualization of the filling material in the canal space. In the present study, cone beam provide indication about the quality of the filling material along the root canal length (longitudinal) as well as different transverse slices of the obturation. Nevertheless, cone beam images lack the resolution, when compared with stereomicroscopic image, in differentiation of sealer from gutta percha. Thus, both non-destructive and destructive methods of 
investigations were used to provide complementary information in the present study. Destructive methods involve sectioning of the filled root canals and examination by stereomicroscope, which is useful for examining the percentage of gutta-perchafilled areas and sealer-filled areas $(1,18,20)$.

The results of the present study showed that gutta core obturation after diode laser produced significantly higher PGFA and lower PSFA $(\mathrm{P}<.05)$ in the curved canals. The possible explanation for the higher percentage of gutta percha of the laser activated irrigation of both EDTA and $\mathrm{NaOCl}$ is oxygen-releasing potential of $\mathrm{NaOCl}$ solution which enhance cavitation formation through generation of water vapor ${ }^{(34)}$ to flow of the gutta-percha to fill the root canal and its irregularities Provides a 3D and thus a maximum amount of gutta percha packed into the canal with a minimal amount of sealer. This is very important for the clinical aspect (20).

The present study used a standardized irrigant protocol for removal of inorganic and organic components of smear layer; $2.5 \% \mathrm{NaOCl}$ and $15 \%$ EDTA, with adequate volume, agitation time and frequent replenishment ${ }^{(16)}$ as recommended earlier. Laser induced cavitation and side-vented needle non-laminar streaming probably provided better mechanical turbulence to penetrate, and effectively carry away the particles ${ }^{(16,27)}$, At the apical level (2 $\mathrm{mm})$, the Gutta core and diode laser group produced significantly higher PGFA and lower PSFA $(\mathrm{P}<.05)$ than all other groups. The Gutta core and EDTA group produced significantly higher PGFA than Single cone and EDTA and Single cone and diode laser $(\mathrm{P}<.05)$.

At the 4-mm level, the Gutta core and diode laser group obturation was superior in terms of PGFA and PSFA compared with all other groups. These findings are in contrast with a previous report (8) but in agreement with several other studies $^{(6,7,22,23)}$. In the latter studies no significant differences between laterally compacted and single-cone gutta-percha were detected at any level ${ }^{(6,7,23)}$, whereas in one study, single-cone obturation produced significantly higher PGFA at the 2-mm level than lateral compaction ${ }^{(22)}$. The present results are also in conformity with other findings that single-cone obturation was able to fill the apical portion of canals effectively, thereby preventing leakage as effectively as vertical and lateral compaction techniques ${ }^{(14,16-18,29)}$. However, the use of single-cone obturation should be limited to round canals because.

\section{CONCLUSIONS}

The results reinforced that 980-nm Diode activated irrigation improved the quality of obturation of the mesial root canals in mandibular molars by using gutta core or single cone. Variation in root canal obturation technique and irrigation activation influenced the percentage of gutta percha filled area and percentage of sealer filled area

\section{REFERENCES}

1- Schäfer E,Schrenker C,Zupanc J, Bürklein S.Percentage of gutta-percha filled areas in canals obturated with crosslinked gutta-percha core-carrier systems, single-cone and lateral compaction technique. J Endod 2016;42(2):294-8.

2- Metzger Z, Teperovich E, Cohen R, Zary R, Paque F, HülsmannM.The Self-adjusting File (SAF). Part 3: Removal of Debris andSmear Layer-A Scanning Electron Microscope StudyJ Endod 2010; 36:697-702.

3. Rodriguez-Figueroa C, McClanahan S B, Bowles W R. Spectrophotometric Determination of Irrigant Extrusion Using Passive Ultrasonic Irrigation, EndoActivator, or Syringe Irrigation. J Endod 2014; 40: 1622-1626.

4. Psimma Z, Boutsioukis C, Kastrinakis E, Vasiliadis L. Effect of needle insertion depth and root canal curvature on irrigant extrusion ex vivo. J Endod 2013;39:521-4.

5. Gu L, Kim J, Ling J, et al. Review of contemporary irrigant agitation techniques and devices. J Endod 2009; 35:791-804.

6. Mancini M, Cerroni L, Iorio L, et al. Smear layer removal and canal cleanliness using different irrigation 
systems (EndoActivator, EndoVac, and passive ultrasonic irrigation): field emission scanning electron microscopic evaluation in an in vitro study. J Endod 2013;39:1456-60.

7. Malki M, Verhaagen B, Jiang LM, et al. Irrigant flow beyond the insertion depth of an ultrasonically oscillating file in straight and curved root canals: visualization and cleaning efficacy. J Endod 2012;38:657-61 .

8. Malentacca A, Uccioli U, Zangari D, et al. Efficacy and safety of various active irrigation devices when used with either positive or negative pressure: an in vitro study. $\mathrm{J}$ Endod 2012;38:1622-6.

9. Layton G, Wu W, Selvaganapathy PR, Friedman S, Kishen A. Fluid dynamics and biofilm removal generated by syringe-delivered and 2 ultrasonic-assisted irrigation methods: a novel experimental approach. J Endod 2015;41:884-889.

10. Ekim SNA, Erdemir A. Comparison of different irrigation activation techniques on smear layer removal: an in vitro study. Microsc. Res. Tech 2015;78:230-239.

11. Deleu E, Meire MA, De Moor RJG. Efficacy of laserbased irrigant activation methods in removing debris from simulated root canal irregularities. Lasers Med Sci 2013;30: 831-835.

12. Guneser MB, Arslan D, Usumez A. Tissue dissolution ability of sodium hypochlorite activated by photoninitiated photoacoustic streaming Technique. J Endod 2015;41:729-732.

13- Da Costa Lima GA, Aguiar CM, Câmara AC , Luiz Carlos Alves LC, dos Santos FAB, do Nascimento AE. Comparison of smear layer removal using the Nd:YAG Laser, ultrasound, ProTaper universal system, and CanalBrush methods: an in vitro Study. J Endod 2015; 41:400-404.

14. Arslan H, Ayranc1 L, Karatas E,Topcuoglu H, Yavuz M, Kesim B .Effect of Agitation of EDTA with 808-Nanometer Diode Laser on Removal of Smear Layer. J Endod 2013;39:1589-1592.

15. Lagemann M, George R, Chai L, Walsh LJ. Activation of ethylenediaminetetraacetic acid by a $940 \mathrm{~nm}$ Diode laser for enhanced removal of smear layer.Aust Endod J 2014; 40: 72-75.

16. Eid $\mathrm{G}$ and Abdelgawad L. Efficacy of $980 \mathrm{~nm}$ diode laser, sonic Endoactivator and side-vented needle agitation techniques on cleanliness of oval-shaped root canals: A scanning electron microscope study. E.D.J 2015; Vol. 61 (3) : 3541-3549.

17. Whitworth J. Methods of filling root canals: principles and practices. Endod Topics 2005;12:2-24.

18. Sch€afer $\mathrm{E}, \mathrm{K} €$ oster $\mathrm{M}, \mathrm{B} €$ urklein $\mathrm{S}$. Percentage of gutta-percha filled areas in canals instrumented with NiTi systems and obturated with matching single cones.J Endod 2013;39:924-8.

19. Soo WK, Thong YL, Gutmann JL. A comparison of four gutta-percha techniques in simulated $\mathrm{C}$-shaped canals. Int Endod J 2015;48:736-46.

20. Jarrett IS, Marx D, Covey D, et al. Percentage of canals filled in apical cross sections: an in vitro study of seven obturated techniques. Int Endod J 2004; 37:392-8.

21. Li GH, Niu LN, Selem LC, et al. Quality of obturation achieved by an endodontic core carrier system with crosslinked gutta-percha carrier in single-rooted canals. J Dent 2014; 42:1124-34.

21. Gordon MP, Love RM, Chandler NP. An evaluation of .06 tapered gutta-percha cones for filling of .06 taper prepared curved root canals. Int Endod J 2005;38:87-96.

22. Sch€afer E, Nelius B, B€urklein S. A comparative evaluation of gutta-percha filled areas in curved root canals obturated with different techniques. Clin Oral Investig 2012; 16:225-30.

23. Yilmaz Z, Deniz D, Ozcelik B, et al. Sealing efficiency of BeeFill 2in1 and System B/ Obtura II versus single-cone and cold lateral compaction techniques. Oral Surg Oral Med Oral Pathol Oral Radiol Endod 2009;108:e51-5.

24. Kontakiotis EG, Wu MK, Wesselink PR. Effect of sealer thickness on long-term sealing ability: a 2-year follow-up study. Int Endod J 1997;30:307-12.

25. Schneider SW. Acomparison of canal preparations in straight and curved root canals. Oral Surg Oral Med Oral pathol 1971;32:271-5.

26. Vertucci FJ. Root canal anatomy of the human permanent teeth . Oral Surf Oral Med Oral path 1984;58; 589-99.

27. Young-Mi Moon, Hyeon-Cheol Kim, Kwang-ShikBae, Seung-Ho Baek,Won-Jun Shon, and WooCheol Lee. Effect of Laser-activated Irrigation of 1320-Nanometer Nd:YAG Laser on Sealer Penetration in Curved Root Canals. J Endod 2012;38:531-535. 
28. Camargo SE, Valera MC, Camargo CH, Fonseca MB, Menezes MM. Effects of Nd:YAG laser irradiation on root canal dentin wall: a scanning electron microscopic study. Photomed Laser Surg 2005;23:399-404.

29. Matsuoka E, Jayawardena JA, Matsumoto K. Amorphological study on root canal preparation using erbium, chromium: YSGG laser. J Oral Laser Appl 2005; 5:17-22.

30. George R, Walsh LJ. Apical extrusion of root canal irrigants when using Er:YAG and Er, Cr:YSGG lasers with optical fibers: an in vitro dye study. J Endod 2008; 34:706-8.

31. Kazemi RB, Safavi KE, Spangberg LSW. Dimensional changes of endodontic sealers. Oral Surg Oral Med Oral Pathol 1993; 76:766-71.
32. Somma F, Cretella G, Carotenuto M, et al. Quality of themoplasticized and single point root fillings assessed by micro-computed tomography. Int Endod J 2011; 44:362-9.

33. Gupta R, Dhin GRa A, Ruchi R. Comparative Evaluation of Three Different Obturating Techniques Lateral Compaction, Thermafil and Calamus for Filling Area and Voids Using Cone Beam Computed Tomography: An Invitro study. Journal of Clinical and Diagnostic Research. 2015 Aug, Vol-9(8): ZC15-ZC17.

34. Hmud R, Kahler WA, George R, Walsh LJ. Cavitational effects in aqueous endodontic irrigants generated by near -infrared lasers .J Endod 2010;36:275-8. 